\title{
Metabolomics study on primary dysmenorrhea patients during the luteal regression stage based on ultra performance liquid chromatography coupled with quadrupole-time-of-flight mass spectrometry
}

\author{
LING FANG, CAIYUN GU, XINYU LIU, JIABIN XIE, ZHIGUO HOU, \\ MENG TIAN, JIA YIN, AIZHU LI and YUBO LI \\ Tianjin State Key Laboratory of Modern Chinese Medicine, School of Chinese Materia Medica, \\ Tianjin University of Traditional Chinese Medicine, Tianjin 300193, P.R. China
}

Received October 13, 2015; Accepted November 2, 2016

DOI: $10.3892 / \mathrm{mmr} .2017 .6116$

\begin{abstract}
Primary dysmenorrhea (PD) is a common gynecological disorder which, while not life-threatening, severely affects the quality of life of women. Most patients with PD suffer ovarian hormone imbalances caused by uterine contraction, which results in dysmenorrhea. PD patients may also suffer from increases in estrogen levels caused by increased levels of prostaglandin synthesis and release during luteal regression and early menstruation. Although PD pathogenesis has been previously reported on, these studies only examined the menstrual period and neglected the importance of the luteal regression stage. Therefore, the present study used urine metabolomics to examine changes in endogenous substances and detect urine biomarkers for PD during luteal regression. Ultra performance liquid chromatography coupled with quadrupole-time-of-flight mass spectrometry was used to create metabolomic profiles for 36 patients with PD and 27 healthy controls. Principal component analysis and partial least squares discriminate analysis were used to investigate
\end{abstract}

Correspondence to: Dr Yubo Li, Tianjin State Key Laboratory of Modern Chinese Medicine, School of Chinese Materia Medica, Tianjin University of Traditional Chinese Medicine, 312 Anshan West Road, Tianjin 300193, P.R. China

E-mail: yuboli1@163.com

Abbreviations: PD, primary dysmenorrhea; UPLC-Q/TOF-MS, ultra performance liquid chromatography quadrupole-time-of-flight mass spectrometry; PCA, principal component analysis; PLS-DA, partial least squares discriminate analysis; ROC, receiver operating characteristic; AUC, area under the curve; TCM, traditional Chinese medicine; QC, quality control; VIP, variable-importance plots; PGE2, prostaglandin E2; PGF2 $\alpha$, prostaglandin F2 $\alpha$

Key words: metabolomics, primary dysmenorrhea, biomarker, luteal regression stage, ultra-performance liquid chromatography quadrupole time-of-flight mass spectrometry the metabolic alterations associated with PD. Ten biomarkers for PD were identified, including ornithine, dihydrocortisol, histidine, citrulline, sphinganine, phytosphingosine, progesterone, 17-hydroxyprogesterone, androstenedione, and 15-keto-prostaglandin F2 $\alpha$. The specificity and sensitivity of these biomarkers was assessed based on the area under the curve of receiver operator characteristic curves, which can be used to distinguish patients with PD from healthy controls. These results provide novel targets for the treatment of PD.

\section{Introduction}

Dysmenorrhea is a common gynecological disorder, occurring during adolescence and throughout reproductive maturity (1). There are two categories; primary and secondary dysmenorrhea. Primary dysmenorrhea (PD) accounts for $>90 \%$ of dysmenorrhea patients and occurs in the absence of other diseases, while secondary dysmenorrhea is caused by a disease of the reproductive organs, such as endometriosis (1). Dysmenorrhea is characterized by spasmodic pain in the hypogastric and lumbar regions between, prior to and during menstruation, with severe patients experiencing fainting (1). According to previous epidemiological studies using multiple survey methods, primary dysmenorrhea occurs in 20-90\% of women $(2,3)$. Although primary dysmenorrhea is not life-threatening, the degree of pain severely affects women's quality of life and ability to work normally. Therefore, the study of clinical treatments for primary dysmenorrhea is of great importance. The luteal regression stage, where the corpus luteum degenerates in the absence of pregnancy, is an important period during the menstrual cycle, and hormonal and endocrinal changes during this time affect the occurrence and development of dysmenorrhea (4).

Metabolomics is a discipline that studies the set of metabolites present at a point in time in vivo, and how surroundings, physiology, pathology and genetic mutations influence the makeup of the metabolome (5). Metabolites are closely associated with phenotypic changes and are often the end result of disease-associated endogenous substance perturbations (6). 
Urine metabolomics is a non-invasive analysis method that is normally used in screening for disease, but has great potential in studies of metabolomics. Ultra performance liquid chromatography coupled with quadrupole-time-of-flight mass spectrometry (UPLC-Q/TOF-MS) is a powerful method of analysis, advantageous due to more faster analyses, shorter time and increased separation efficiency compared with other traditional instruments $(7,8)$. Due to this high throughput, high sensitivity and high accuracy, it is possible to dynamically analyze urine to identify and resolve metabolic differences in endogenous substances in vivo. The results can provide clinical guidance in the study of disease mechanisms.

Previous studies have primarily been concerned with researching menstruation biomarkers (9). To the best of our knowledge, the mechanisms underlying dysmenorrhea during the luteal regression stage have not yet been reported, making research on the luteal regression stage mechanisms of PD urgently required. Therefore, the changes in endogenous substances during the luteal regression stage were studied, using urine metabolomics combined with receiver operator characteristic (ROC) curves to verify biomarkers. Initially, a UPLC-Q/TOF-MS technique was used to generate a metabolomic profile of urine samples from patients with PD, as well as healthy controls. Principal component analysis (PCA) and partial least squares discriminate analysis (PLS-DA) were used to identify metabolite perturbations. Next, the classification performance (specificity and sensitivity) was verified using the area under the curve (AUC) of the ROC curves. The present study aimed to confirm the endogenous metabolites present at the luteal regression stage and seek potential PD biomarkers from among them. The results of this may assist in the clinical treatment of PD.

\section{Materials and methods}

Study subjects and design. The present study was reviewed and approved by the Tianjin University of Traditional Chinese Medicine. The samples used in the present study originated from the same volunteers used by Fang et al (9), and so shared demographic and clinical characteristics. The group included 36 patients with clinically diagnosed PD and 27 healthy controls from the Affiliated Hospital of Tianjin University of Chinese Medicine (Tianjin, China) and Tianjin Maternity Hospital (Tianjin, China). Urine was collected from PD patients and healthy controls during the luteal regression stage, and informed consent was obtained from all participants prior to sample collection. Each volunteer provided written answers detailing age, weight, height, personal and family history of menstrual cramps, and dysmenorrhea pain integral. The pain integral was $>8$ in all patients with PD. The selection criteria for patients with PD were in accordance with the diagnostic criteria established by the People's Republic of China Ministry of Health Pharmaceutical Council (10) and the National Higher School Teaching Materials Obstetrics and Gynecology, Seventh Edition (11).

Collection of urine samples. Urine samples were collected as described previously (9), and collection was consistent with the clinical inclusion criteria (morning urine collected three days prior to menstruation, and the volume of each sample recorded). Following this, each sample was loaded into $10 \mathrm{ml}$ centrifuge tube, and $1 \%$ sodium azide was added. The samples were subjected to centrifugation at $765 \times \mathrm{g}$ at $4^{\circ} \mathrm{C}$ for $15 \mathrm{~min}$. The supernatant was stored at $-80^{\circ} \mathrm{C}$ until analysis.

Urine sample preparation. Urine samples and quality control (QC) samples were prepared as previously described (9). QC samples were mixture of urine from PD patients and healthy controls. Urine samples were prepared as follows: Samples were thawed at room temperature and centrifuged at 8,497 $\mathrm{x} g$ at $4^{\circ} \mathrm{C}$ for $10 \mathrm{~min}$. Next, $200 \mu \mathrm{l}$ liquid supernatant was added to $200 \mu \mathrm{l}$ pure water. The liquid was subsequently vortexed for $1 \mathrm{~min}$ and centrifuged at $14,360 \times \mathrm{g}$ at $4^{\circ} \mathrm{C}$ for $15 \mathrm{~min}$. Finally, all samples were injected into UPLC-Q/TOF mass spectrometer for analysis. QC samples were prepared as follows: 8 samples were selected from each group by random sampling. QC samples were centrifuged at $8,497 \times g$ at $4^{\circ} \mathrm{C}$ for $10 \mathrm{~min}$. Liquid supernatant $(200 \mu \mathrm{l})$ was then added to $200 \mu \mathrm{l}$ pure water, vortexed for $1 \mathrm{~min}$ and centrifuged at 14,360 x $g$ at $4^{\circ} \mathrm{C}$ for $15 \mathrm{~min}$. QC samples were analyzed once every $6 \mathrm{~h}$ and used to test instrument stability, ensuring that conditions remained the same throughout the analysis.

UPLC-Q/TOF-MS analysis. The liquid chromatograph used was the Waters ACQUITY UPLC system (Waters Corporation, Milford, MA, USA). Supernatant (5 $\mu \mathrm{l})$ was injected into ACQUITY UPLC BEH C18 columns $(2.1 \mathrm{~mm}$ x $100 \mathrm{~mm}$; $1.7 \mu \mathrm{m}$; Waters Corporation). The column temperature was set

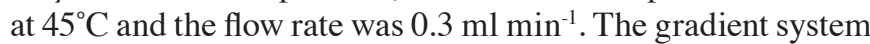
consisted of mobile phase A ( $0.1 \%$ formic acid in water) and mobile phase $\mathrm{B}(0.1 \%$ formic acid in acetonitrile) as follows: 0-8.5 min, 1-25\% B; 8.5-11 min, 25-50\% B; 11-13 min, 50-90\% B; $13-15 \min , 90-99 \% \mathrm{~B} ; 15-17 \mathrm{~min}, 99 \% \mathrm{~B}$; 17-18.5 min, 99-1\% B; 18.5-20 min, 1\% B (9).

Mass spectrometry was performed on a Waters Micromass QTOF Micro Synapt high definition mass spectrometer (Waters Corporation). Electrospray ionization (ESI) was used in positive mode. Ion source parameters were as follows: Capillary voltage, $3.0 \mathrm{kV}$; cone voltage, $30 \mathrm{~V}$; nebulizer pressure, $350 \mathrm{psi}$; nitrogen gas temperature, $325^{\circ} \mathrm{C}$; cone gas flow, $50 \mathrm{l} / \mathrm{h}$; desolvation gas flow, $600 \mathrm{l} / \mathrm{h}$; source temperature, $120^{\circ} \mathrm{C}$; desolvation temperature, $350^{\circ} \mathrm{C}$; $0.1 \mathrm{sec}$ (interval $0.02 \mathrm{sec}$ ) collected once spectrum data and scanned at mass range of $\mathrm{m} / \mathrm{z} 50-1,100$.

Data processing and multivariate analysis. Data was initially exported using MarkerLynx V4.1 (Waters Corporation) with peak discovery, peak alignment and raw data filtering to determine potential discriminant variables. A data array that included retention time, $\mathrm{m} / \mathrm{z}$ values and normalized peak area was then obtained. The multivariate data matrix was exported into SIMCA-P 12.0 software (MKS Instruments, Andover, MA, USA) for PCA and PLS-DA analysis. The PCA model is a statistical sample of the principal contradiction reaction and can resolve the main factor multivariate data, as well as reflect the main features. The data space is compressed, and characteristics of multivariate data in a low-dimensional space are expressed through visual effects. The PLS-DA model was fitted following the adaptation of the data. According to the classification pattern recognition model, compounds 
were chosen depending on whether they have an important contribution. These data further validate the differences in the compounds between PD patients and healthy controls through metabolic differences in the luteal regression stage $(12,13)$. Cross-validation tests were sorted through for verification. Evaluating urine PLS-DA models requires a higher $R^{2} Y$ value, and $Q^{2}$ values for these parameters typically. $R^{2} X, R^{2} Y$ and $Q^{2}$ are larger in the present model, and the $\mathrm{R}^{2} \mathrm{Y}$ and $\mathrm{Q}^{2}$ are closer to 1 , demonstrating the accuracy of the model result (14). Variable-importance plots (VIP) values $>1$ were used to screen potential biomarkers. Unpaired Student's $t$-tests were used to determine statistically significant differences between biomarkers in patients with $\mathrm{PD}$ and healthy controls. $\mathrm{P}<0.05$ was considered to indicate a statistically significant difference.

Resulting candidate biomarkers were selected based on their molecular weights and use of the formula to predict the elemental composition of a compound. The database was then used to retrieve documents and metabolite candidates, and the candidates were finally confirmed using metabolic standards, references and tandem mass spectrometry information, such as the Human Metabolome Database (HMDB; http://www. hmdb.ca/) (15-17) and MassBank (http://www.massbank.jp/). Related metabolites were submitted to a correlation analysis of metabolic pathways associated with metabolic pathway analysis (MetPA) (18) and the Kyoto Encyclopedia of Genes and Genomes (KEGG; http://www.genome.jp/kegg/) (19).

\section{Results}

Metabolomic profiling analysis. Urinary metabolic fingerprints of patients with primary dysmenorrhea ( $\mathrm{T}$ group) and healthy controls ( $Z$ group) during the luteal regression stage are displayed in Fig. 1. Several different peak intensities were clearly detected from the typical base peak intensity (BPI), which indicates two sets of data with metabolite differences in Fig. 1.

Data were processed using multivariate statistical analysis methods (Fig. 2). In the PCA scatter plot, each point represents a volunteer sample, making it possible to visually discern the differences between the samples. In addition, anomalous samples can be identified and removed $(20,21)$ to improve the accuracy of the model. PLS-DA (Fig. 2A) was used to identify the metabolites that differentiate patients with PD from the healthy controls. The $\mathrm{R}^{2} \mathrm{Y}$ and $\mathrm{Q}^{2}$ of the PLS-DA model were 0.992 and 0.806 , respectively, demonstrating the accuracy of the model. Finally, a VIP value $>1$ and unpaired Student's $t$-tests were used to identify metabolites with significant differences as potential biomarkers. Following this, a score plot (S-plot) of the PLS-DA was used to identify potential discriminatory metabolites (Fig. 2B). Metabolites that are distal from the origin and close to the vertical axis of the S-plot are the differentiating metabolites.

Identification of biomarkers. PLS-DA analysis VIP values with $>1$ ion were used to identify candidate biomarkers. Significantly different endogenous compounds during the luteal regression stage were considered to be the differentiating compounds between patients with PD and healthy controls, resulting in the identification of 10 specific biomarkers. Levels of citrulline, ornithine, androstenedione, progesterone, phytosphingosine, dihydrocortisol and 17-hydroprogesterone were significantly decreased in patients with PD compared with healthy controls during the luteal regression stage $(\mathrm{P}=0.0426$, $\mathrm{P}=0.0071, \mathrm{P}=0.0040, \mathrm{P}=0.0359, \mathrm{P}=0.0360, \mathrm{P}=0.0454$ and $\mathrm{P}=0.0235$, respectively; Table I) and levels of sphinganine, histidine and 15-keto-prostaglandin F2 $\alpha$ were significantly increased in patients with PD compared with healthy controls during the luteal regression stage $(\mathrm{P}=0.0136, \mathrm{P}=0.0107$ and $\mathrm{P}=0.0001$, respectively; Table I).

Metabolite $\mathrm{m} / \mathrm{z}$ values were used to determine the probable molecular formula using the HMDB database (15-17). Of these compounds, 4 were identified using authentic standards and 6 were identified by comparing the fragments based on their molecular ion information and MS/MS data. An example is 17-hydroxyprogesterone, which exhibited an accurate biomarker mass $\left([\mathrm{M}+\mathrm{H}]^{+}\right.$at $\left.\mathrm{m} / \mathrm{z} 331.2241\right)$ in the mass spectrum. In positive ion mode, the MS/MS contains the fragment ions $\mathrm{m} / \mathrm{z} 195.1\left[\mathrm{M}+\mathrm{H}-\mathrm{C}_{9} \mathrm{H}_{12} \mathrm{O}\right]^{+}$and $\mathrm{m} / \mathrm{z} 138.0$ $\left[\mathrm{M}+\mathrm{H}-\mathrm{C}_{12} \mathrm{H}_{17} \mathrm{O}_{2}\right]^{+}$. The HMDB database was also used to confirm the results (15-17).

Biomarker verification. To determine the sensitivity and specificity of the 10 biomarkers identified in the luteal regression stage, SPSS 17.0 (SPSS Inc., Chicago, IL, USA) was used to analyze the ROC curves of the biomarkers. The ROC curves were regarded as a potential diagnosis threshold for the test results, and the sensitivity and specificity were calculated and evaluated. Data were plotted as 1-specificity on the $x$ axis and sensitivity on the $y$ axis (Fig. 3). The ROC curve is proximal to the upper left (Fig. 3B, blue line), and the largest point boundary value of the Youden index (22) is the threshold. Therefore, the sensitivity and specificity of the test are greater, and the rates of misdiagnoses and missed diagnoses are relatively low. With regard to the plotted curve and $45^{\circ}$ oblique linear contrast, if most of the curve coincides with the independent variable, the value is a poor predictive value for the dependent variable. If the data curves away from the $45^{\circ}$ oblique line more than the independent variable, the value is a better predictive value for the dependent variable. In the present study the biomarkers with AUC $>0.7$ were considered to have an important role in patients with PD. Finally, as demonstrated in (Fig. 3A), the AUC of 6 metabolites was $>0.8$. Thus, 6 important biomarker candidates were identified. The combination of the 10 biomarkers' AUC at the $95 \%$ confidence interval was 0.950 , indicating high sensitivity and specificity during the luteal regression stage (Fig. 3B).

Metabolic pathways. MetPA (18), which uses a library of metabolic pathways from the KEGG (19), was used for metabolic pathway analyses of potential biomarkers (Fig. 4). The abscissa is the impact of the pathways. Where the pathway impact value calculated from the pathway topology analysis is $>0$, that pathway is likely associated with PD. It was revealed that steroid hormone biosynthesis, sphingolipid metabolism, arginine and proline metabolism, histidine metabolism and arachidonic acid metabolism were perturbed at the luteal regression stage in patients with PD (Fig. 4). Finally, the disturbed metabolic pathways detected by UPLC-Q/TOF-MS analysis were analyzed (Fig. 5). 


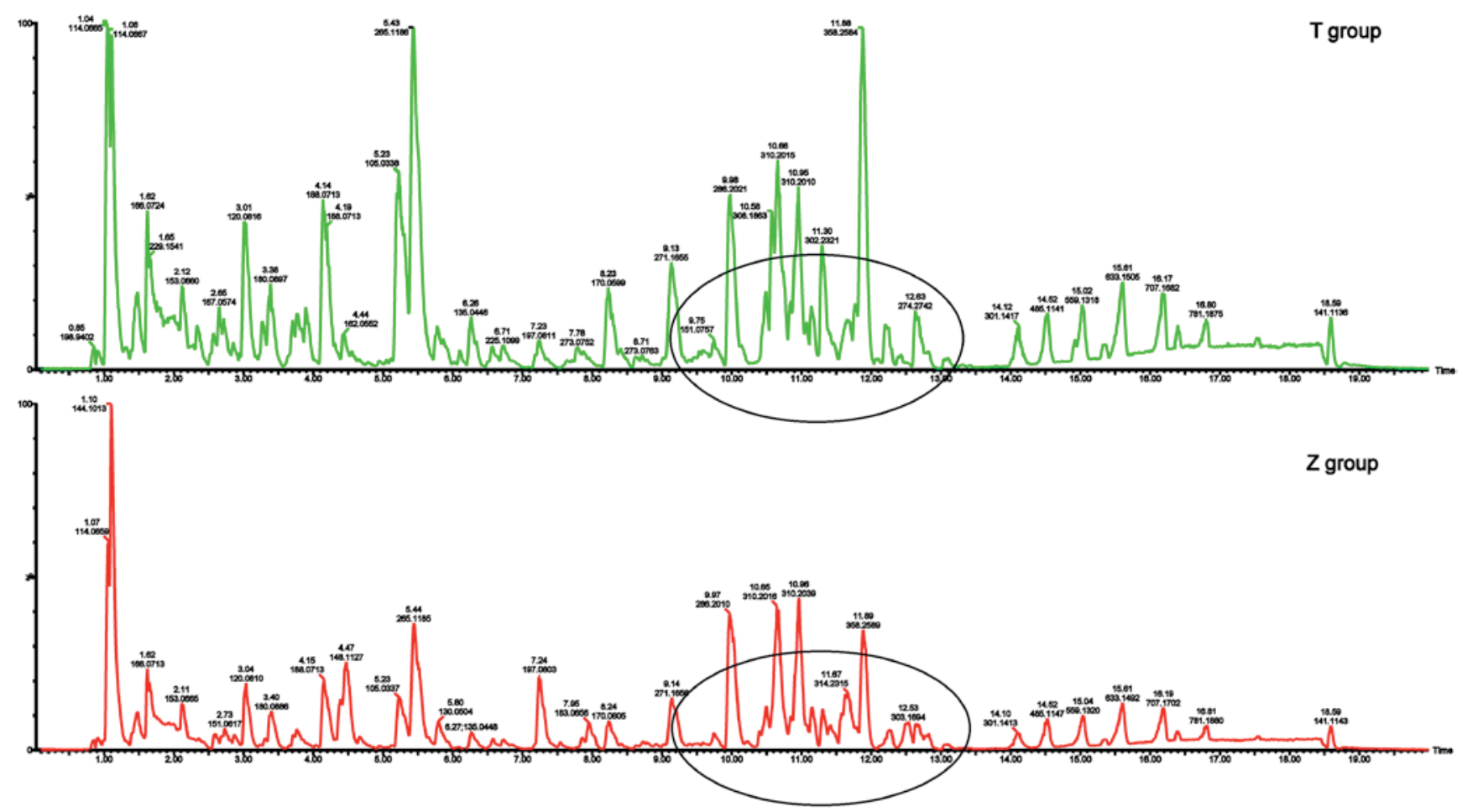

Figure 1. Typical base peak intensity chromatogram of urine for primary dysmenorrhea patients $(\mathrm{T})$ and healthy controls $(\mathrm{Z})$ at positive electrospray ionization mode. Differences in metabolites are indicated.

A

- Z group

- T group

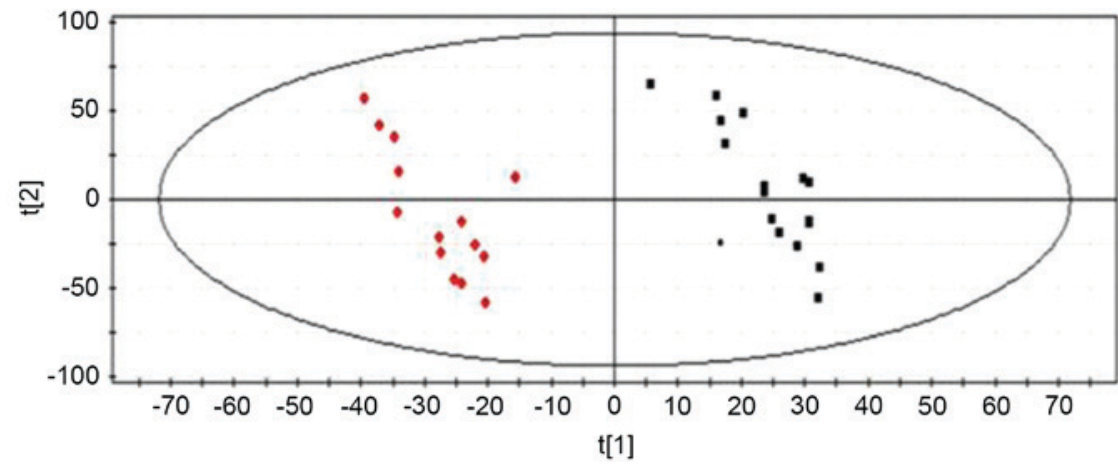

B OPLS/O2PLS-DA

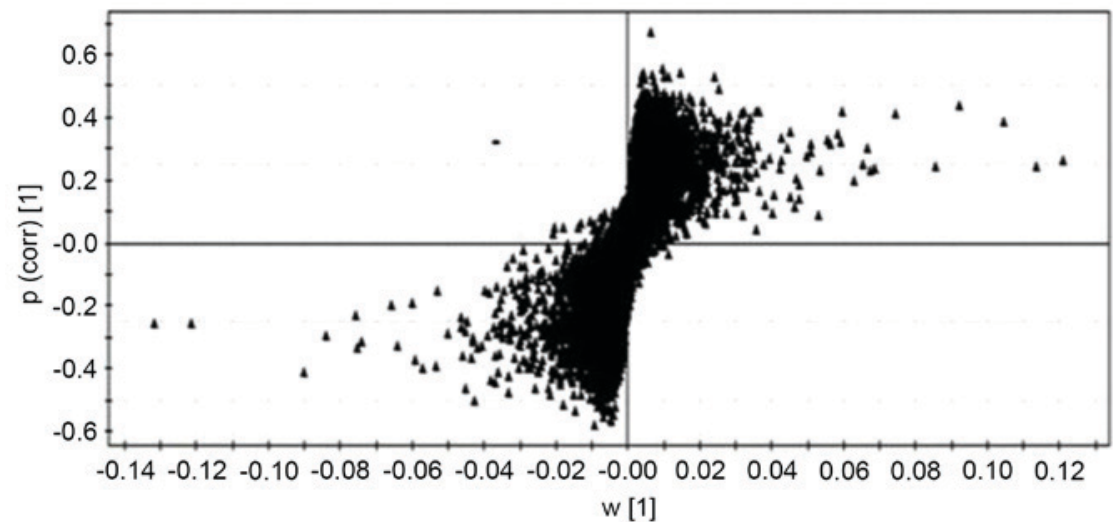

Figure 2. Multivariate statistical analysis. (A) PLS-DA model of ultra performance liquid chromatography coupled with quadrupole-time-of-flight mass spectrometry data between patients with PD and healthy controls in the luteal regression stage in positive mode. (B) S-plot of PLS-DA model between patients with PD and healthy control. PLS-DA, partial least squares discriminate analysis; PD, primary dysmenorrhea. 

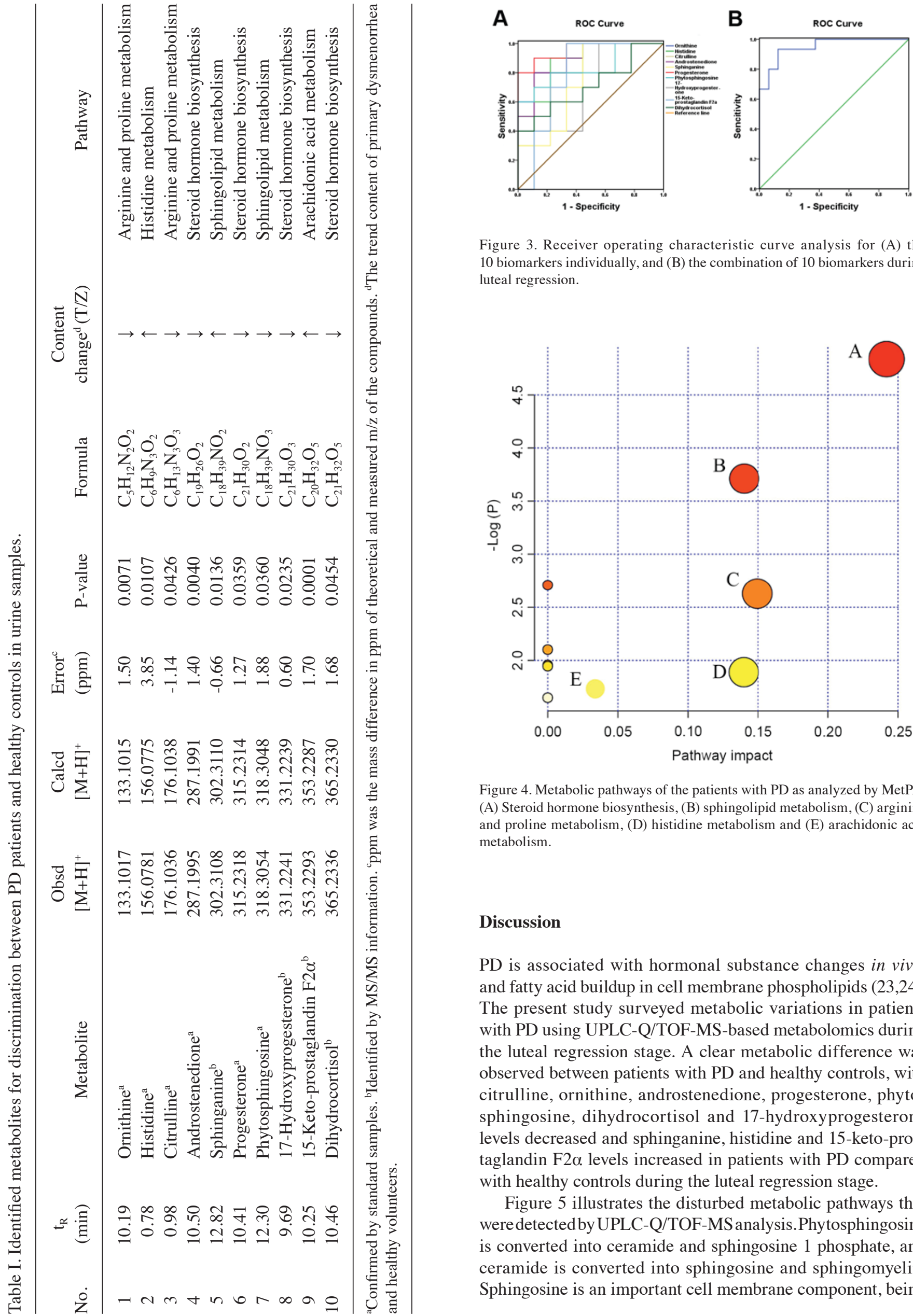

Figure 3. Receiver operating characteristic curve analysis for (A) the 10 biomarkers individually, and (B) the combination of 10 biomarkers during luteal regression.

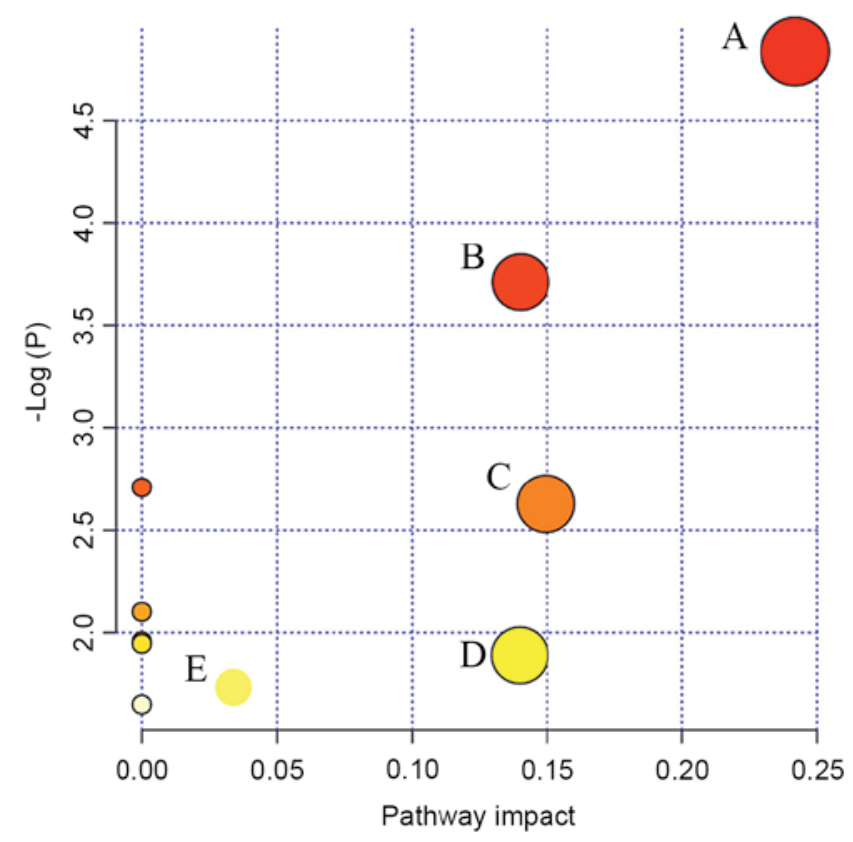

Figure 4. Metabolic pathways of the patients with PD as analyzed by MetPA. (A) Steroid hormone biosynthesis, (B) sphingolipid metabolism, (C) arginine and proline metabolism, (D) histidine metabolism and (E) arachidonic acid metabolism.

\section{Discussion}

PD is associated with hormonal substance changes in vivo, and fatty acid buildup in cell membrane phospholipids $(23,24)$. The present study surveyed metabolic variations in patients with PD using UPLC-Q/TOF-MS-based metabolomics during the luteal regression stage. A clear metabolic difference was observed between patients with PD and healthy controls, with citrulline, ornithine, androstenedione, progesterone, phytosphingosine, dihydrocortisol and 17-hydroxyprogesterone levels decreased and sphinganine, histidine and 15-keto-prostaglandin F2 $\alpha$ levels increased in patients with PD compared with healthy controls during the luteal regression stage.

Figure 5 illustrates the disturbed metabolic pathways that were detected by UPLC-Q/TOF-MS analysis.Phytosphingosine is converted into ceramide and sphingosine 1 phosphate, and ceramide is converted into sphingosine and sphingomyelin. Sphingosine is an important cell membrane component, being 


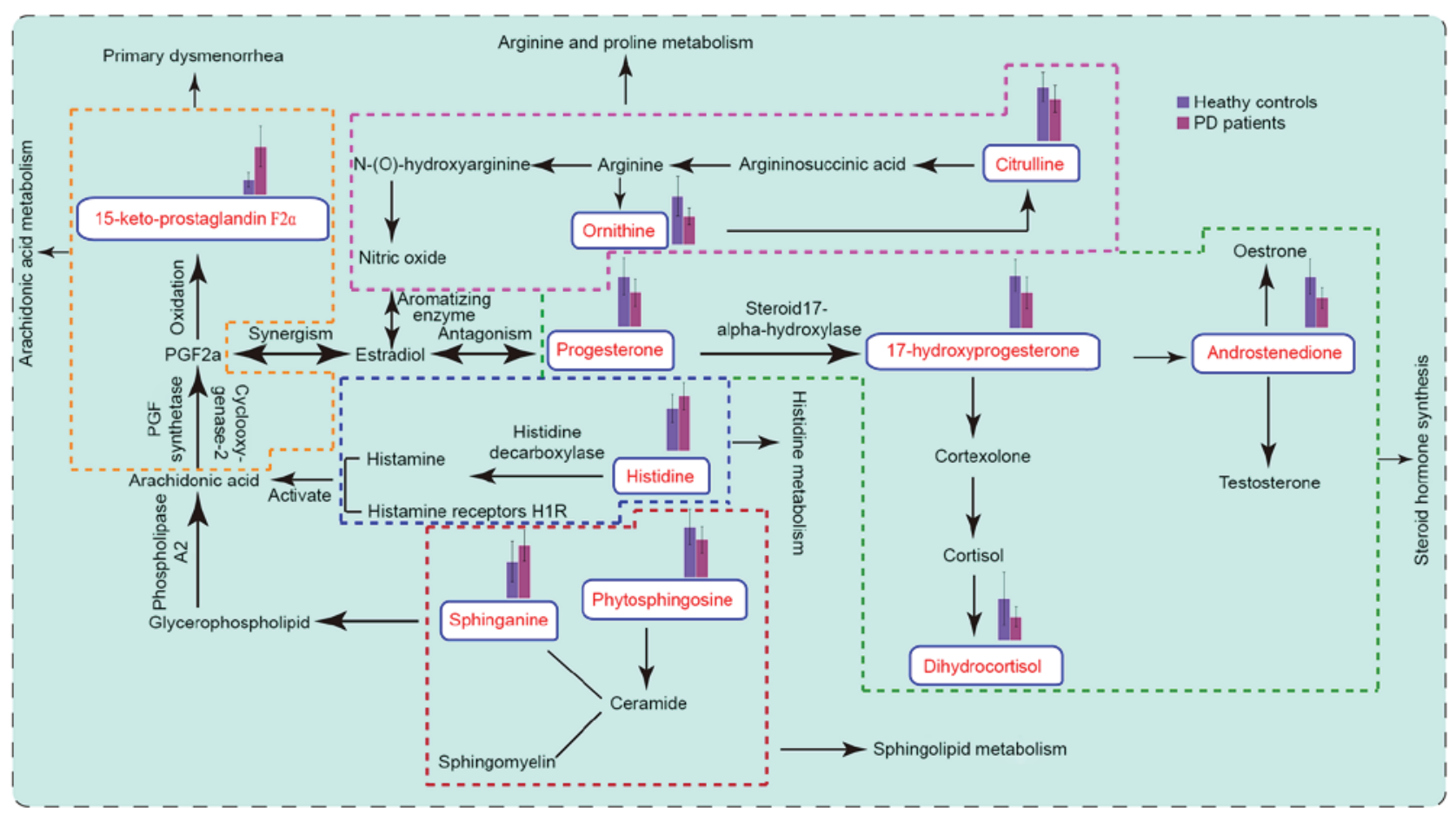

Figure 5. Schematic diagram of the disturbed metabolic pathways detected by ultra performance liquid chromatography coupled with quadrupole-time-of-flight mass spectrometry analysis. Words in red represent the perturbed metabolites of primary dysmenorrhea during luteal regression. Metabolites written in black were not detected but are relevant for all metabolic pathways. The dotted line denotes the five metabolic pathways. The histograms display the increase and decrease in urinary levels.

a nerve receptor ligand, a signal transduction effect factor and, in addition, involved in the binding of prostaglandin E2 (PGE2) and prostaglandin F2 $\alpha$ (PGF2 $\alpha$ ) to the appropriate receptor during signal transduction processes $(25,26)$. Phospholipids in the cell membrane are converted into arachidonic acid by phospholipase A2. Following this, cyclic oxidase and PGF synthase generate PGF $2 \alpha$. PGF $2 \alpha$ has a strong vasoconstrictory effect, and is involved in the contraction of uterine smooth muscle. Furthermore, 15-ketone-prostaglandin F2 $\alpha$ is a PGF $2 \alpha$ metabolite, and the level of 15-ketone-prostaglandin F2 $\alpha$ was increased in patients with PD. This may lead to uterine contractions, resulting in dysmenorrhea during the luteal regression stage. In addition, increased prostaglandin levels may increase peripheral nerve pain perception $(27,28)$.

Progesterone, androstenedione, dihydrocortisol and 17-hydroxyprogesterone are the metabolic products of steroids, which are a class of bioactive compounds derived from cholesterol. Cholesterol is converted into pregnenolone, which is converted into progesterone by mitochondrial enzymes and isomerases. Initially, progesterone uses 17-hydroxylase to form 17-hydroxyprogesterone, and 17-hydroxyprogesterone is converted into androstenedione by carbon chain lyase. Previous studies have demonstrated that PGF2 $\alpha$ levels in the uterine muscle layer positively correlate with estradiol levels and negatively correlate with progesterone levels in the uterine vein. Increased estradiol levels accelerate PGF2 $\alpha$ synthesis and release, as well as cause uterine blood vessel spasms and thus dysmenorrhea, but progesterone antagonizes this reaction (29). During luteal regression, progesterone levels in patients with PD was significantly lower than in the healthy control, and estradiol levels were increased. It has previously been reported that the level of progesterone decreased in mice when the animal's pain threshold decreased, but no pain was detected when progesterone secretion levels were high (30). In addition, dihydrocortisol is involved in cortisol metabolism, and in vitro experiments have demonstrated that cortisol increases the prostaglandin levels in uterine smooth muscle tissue and increases myometrial contractions (31). The results of the present study also suggest that changes in prostaglandin levels may induce dysmenorrhea during luteal regression.

Histidine is converted to histamine by histamine decarboxylase. Histamine is a metabolite in mast cells and typically exists in an inactive binding state. Mast cells exist in the female reproductive system and are widely distributed in the human uterine muscle layer. Histamine indirectly regulates normal uterine smooth muscle contraction through activation of its receptors $(32,33)$. Previous studies indicate that activation of the histamine receptor increases pain sensitivity in mice (34). An increase in histidine content leads to an increase in histamine content, which increases uterine smooth muscle contractions and resulted in dysmenorrhea (34).

Arginine is the precursor of nitric oxide (NO) and ornithine, which are involved in citrulline synthesis. Nitric oxide is a neurotransmitter involved in modulating peripheral and central pain level, and is important for nervous and immune system regulation. Through the NO-cyclic guanosine monophosphate pathway, NO induces both pain and analgesic effects: When reduced, NO induces pain and results in dysmenorrhea, but when increased it inhibits the induced 
pain. During luteal regression, ornithine and citrulline are involved in arginine metabolism (35). When ornithine and citrulline levels were reduced, nitric oxide content was reduced and its analgesic effect suppressed, resulting in dysmenorrhea (36). Therefore, in future studies, drug interventions targeting these metabolic pathway biomarkers will be used to alleviate pain, to reduce the distress caused by PD and to discern the optimal time of administration.

By comparing PD biomarkers between menstruation and luteal regression in patients with PD, it was revealed that primary dysmenorrhea patients are affected by perturbations to steroid metabolic pathways during the menstrual period and luteal regression stage (9). The hypothalamus-pituitary-gonadal axis is important for hormone regulation during the menstrual cycle (37). The main ovarian hormones, estrogen and progesterone, regulate menstruation (38). Progesterone is able to promote estradiol transformation into low active estrone, thereby generating reduced prostaglandin, which consequently reduces the extent of uterine smooth muscle contraction and alleviates dysmenorrhea. In the present study, it was observed that the progesterone levels decreased, and also possibly that the prostaglandin levels increased, which were consistent with previous reports (39). Estrogen is able to stimulate PGF $2 \alpha$ and vasopressin synthesis, which is released in the uterine spiral artery walls, mediated via PGF $2 \alpha$ receptors. PGF $2 \alpha$, in combination with its receptor, leads to the contraction of local blood vessels, and reduced blood flow to the uterus and muscle, due to pain-induced ischemia/hypoxia. Similarly, vasopressin causes the muscle layers of small blood vessels of the uterus to contract, leading to uterine ischemia and pain (27). If a target biomarker is converted to a PD-associated form during luteal regression, and a drug targeting this biomarker is applied during luteal regression, PD may be attenuated. If this occurs, pain in patients with PD may be reduced during menstruation (40). Therefore, discovering these important biomarkers in the luteal regression stage is essential for treating PD.

To conclude, in the present study a UPLC-Q/TOF-MS analysis was used to create metabolic profiles of urine samples during luteal regression, and indicated that metabolomic profiles of patients with PD deviated from the healthy controls. In total, 10 biomarkers were identified in patients with PD during luteal regression, associated with sphingolipid metabolism, steroid hormone biosynthesis and arginine and proline metabolism. ROC curves were used to evaluate biomarker sensitivities and specificities during luteal regression. These results are consistent with the theory of traditional Chinese medicine (TCM) syndrome differentiation, and may help provide novel targets for the treatment of PD.

\section{Acknowledgements}

The present study was supported by The Research Programs of Application of Basic and Frontier Technology in Tianjin (grant no. 13JCYBJC23900), Tianjin University Undergraduates Teaching Quality and Teaching Reform Project (grant no. B07-1008) and the Program for Changjiang Scholars and Innovative Research Team in University (grant no. IRT_14R41).

\section{References}

1. French L: Dysmenorrhoea. Am Fam Physician 71: 285-291, 2005.

2. Banikarim C, Chacko MR and Kelder SH: Prevalence and impact of dysmenorrhea on Hispanic female adolescents. Arch Pediatr Adolesc Med 154: 1226-1229, 2000.

3. Davis AR and Westhoff CL: Primary dysmenorrhea in adolescent girls and treatment with oral contraceptives. J Pediatr Adolesc Gynecol 14: 3-8, 2001.

4. Wallace M, Hashim YY, Wingfield M, Culliton M, McAuliffe F, Gibney MJ and Brennan L: Effects of menstrual cycle phase on metabolomic profiles in premenopausal women. Hum Reprod 25: 949-956, 2010.

5. Nicholson JK and Lindon JC: Systems biology: Metabonomics. Nature 455: 1054-1056, 2008.

6. Vellodi A: Lysosomal storage disorders. Br J Haematol 128 : 413-431, 2005

7. Plumb RS, Johnson KA, Rainville P, Smith BW, Wilson ID, Castro-Perez JM and Nicholson JK: UPLC/MSE; a new approach for generating molecular fragment information for biomarker structure elucidation. Rapid Commun Mass Spectrom 20: 1989-1994, 2006

8. Michopoulos F, Lai L, Gika H, Theodoridis G and Wilson I: UPLC-MS-based analysis of human plasma for metabonomics using solvent precipitation or solid phase extraction. J Proteome Res 8: 2114-2121, 2009.

9. Fang L, Dai N, Wang L, Zhang XX, Liu XY, Wang YM and Li YB: Urine metabolomic study of primary dysmenorrhea patients during menstrual period using an ultra performance liquid chromatography coupled with quadrupole-time-of-flight mass spectrometry (UPLC-Q-TOF-MS). RSC Adv 4: 44208-44213, 2014.

10. People's Republic of China Ministry of Health Pharmaceutical Council: Chinese medicine treatment of dysmenorrhea clinical research guidelines. The National Ministry of Health, China, pp263-266, 1993.

11. Yue $\mathbf{J}$ (ed): Obstetrics and Gynecology. Vol 318. 7th edition, People's Health Publishing House, China, 2008.

12. Wang X, Wang H, Zhang A, Lu X, Sun H, Dong H and Wang P: Metabolomics study on the toxicity of aconite root and its processed products using ultraperformance liquid-chromatography/electrospray-ionization synapt high-definition mass spectrometry coupled with pattern recognition approach and ingenuity pathways analysis. J Proteome Res 11: 1284-1301, 2012.

13. Wang X, Zhang A, Wang P, Sun H, Wu G, Sun W, Lv H, Jiao G, $\mathrm{Xu} \mathrm{H}$, Yuan Y, et al: Metabolomics coupled with proteomics advancing drug discovery toward more agile development of targeted combination therapies. Mol Cell Proteomics 12: 1226-1238, 2013

14. Legido-Quigley C, Stella C, Perez-Jimenez F, Lopez-Miranda J, Ordovas J, Powell J, van-der-Ouderaa F, Ware L, Lindon JC, Nicholson JK and Holmes E: Liquid chromatography-mass spectrometry methods for urinary biomarker detection in metabonomic studies with application to nutritional studies. Biomed Chromatogr 24: 737-743, 2010.

15. Wishart DS, Tzur D, Knox C, Eisner R, Guo AC, Young N, Cheng D, Jewell K, Arndt D, Sawhney S, et al: HMDB: The human metabolome database. Nucleic Acids Res 35 (Database issue): D521-D526, 2007.

16. Wishart DS, Knox C, Guo AC, Eisner R, Young N, Gautam B, Hau DD, Psychogios N, Dong E, Bouatra S, et al: HMDB: A knowledgebase for the human metabolome. Nucleic Acids Res 37 (Database issue): D603-D610, 2009.

17. Wishart DS, Jewison T, Guo AC, Wilson M, Knox C, Liu YF, Djoumbou Y, Mandal R, Aziat F, Dong E, et al: HMDB 3.0-The human metabolome database in 2013. Nucleic Acids Res 41 (Database issue): D801-D807, 2013.

18. Xia J and Wishart DS: MetPA: A web-based metabolomics tool for pathway analysis and visualization. Bioinformatics 26: 2342-2344, 2010.

19. Kanehisa M, Sato Y and Morishima K: BlastKOALA and GhostKOALA: KEGG tools for functional characterization of genome and metagenome sequences. J Mol Biol 428: 726-731, 2016.

20. Rousseeuw PJ and Hubert M: Robust statistics for outlier detection. WIREs Data Mining and Knowledge Discovery 1: 73-79, 2011.

21. Huberta M, Rousseeuwb PJ and Branden KV: ROBPCA: A new approach to robust principal component analysis. Technometrics 47: 64-79, 2005. 
22. Kumar R and Indrayan A: Receiver operating characteristic (ROC) curve for medical researchers. Indian Pediatr 48: 277-287, 2011.

23. Marsh EE, Shaw ND, Klingman KM, Tiamfook-Morgan TO, Yialamas MA, Sluss PM and Hall JE: Estrogen levels are higher across the menstrual cycle in African-American women compared with Caucasian women. J Clin Endocrinol Metab 96: 3199-3206, 2011.

24. Cornel KM, Kruitwagen RF, Delvoux B, Visconti L, Van de Vijver KK, Day JM, Van Gorp T, Hermans RJ, Dunselman GA and Romano A: Overexpression of $17 \beta$-hydroxysteroid dehydrogenase type 1 increases the exposure of endometrial cancer to 17ß-estradiol. J Clin Endocrinol Metab 97: E591-E601, 2012.

25. Arulkumaran S, Kandola MK, Hoffman B, Hanyaloglu AC, Johnson MR and Bennett PR: The roles of prostaglandin EP 1 and 3 receptors in the control of human myometrial contractility. J Clin Endocrinol Metab 97: 489-498, 2012.

26. Li S, Zhang ZQ, Wu LJ, Zhang XG, Li YD and Wang YY: Understanding ZHENG in traditional Chinese medicine in the context of neuro-endocrine-immune network. IET Syst Biol 1: 51-60, 2007.

27. Su S, Duan J, Wang P, Liu P, Guo J, Shang E, Qian D, Tang Y and Tang Z: Metabolomic study of biochemical changes in the plasma and urine of primary dysmenorrhea patients using UPLC-MS coupled with a pattern recognition approach. J Proteome Res 12 : $852-865,2013$

28. Harel Z: A contemporary approach to dysmenorrhea in adolescents. Pediatric Drugs 4: 797-805, 2002.

29. Xue Z, Liu CZ, Gao SZ and Ma YX: The herbal-partitioned moxibustion for primary dysmenorrhea and it's impact on reproductive endocrinal function of patients. Zhongguo Zhen Jiu 34: 209-212, 2014 (In Chinese).

30. Sternberg WF, Mogil JS, Kest B, Page GG, Leong T, Yam V and Liebeskind JC: Neonatal testosterone exposure influences neurochemistry of non-opioid swim stress induced analgesia in adult mice. Pain 63: 321-326, 1995.
31. Morimoto $\mathrm{K}$ and Oku M: Effect of progesterone, cortisol and dehydroepiandrosterone-sulfate on prostaglandin production by cultured human myometrial cells. Nihon Sanka Fujinka Gakkai zasshi 47: 391-397, 1995 (In Japanese).

32. Brown RE, Steven DR and Haas HL: The physiology of brain histamine. Prog Neurobiol 63: 637-672, 2001

33. He S, McEuen AR, Blewett SA, Li P, Buckley MG, Leufkens P and Walls AF: The inhibition of mast cell activation by neutrophil lactoferrin: Uptake by mast cells and interaction with tryptase, chymase and cathepsin G. Biochem Pharmacol 65: 1007-1015, 2003.

34. Mobarakeh JI, Sakurada S, Katsuyama S, Kutsuwa M, Kuramasu A, Lin ZY, Watanabe T, Hashimoto Y, Watanabe T and Yanai K: Role of histamine $\mathrm{H}(1)$ receptor in pain perception: A study of the receptor gene knockout mice. Eur J Pharmacol 391: 81-89, 2000.

35. Yallampalli C, Izumi H, Byamsmith $\mathrm{M}$ and Garfield RE: An L-arginine-nitric oxide-cyclic guanosine monophosphate system exists in the uterus and inhibits contractility during pregnancy. Am J Obstet Gynecol 170: 175-185, 1994.

36. Bodelsson G, Sjöbery NO and Stjernquist M: Contractile effect of endothelin in the human uterine artery and autoradiographic localization of its binding sites. Am J Obstet Gynecol 167: 745-750, 1992.

37. Roozendaal MM: Stress and the hypothalamus-pituitary-gonadal axis in the cyclic rat. Stress \& the Hypothalamus Pituitary Gonadal Axis in the Cyclic Rat 136, 1997.

38. Sherman BM and Korenman SG: Hormonal characteristics of the human menstrual cycle throughout reproductive life. J Clin Invest 55: 699-706, 1975.

39. Rani M, Singh U, Agrawal GG, Natu SM, Kala S, Ghidiyal A and Srivastava N: Impact of yoga nidra on menstrual abnormalities in females of reproductive age. J Altern Complement Med 19: 925-929, 2013.

40. Dawood MY: Primary dysmenorrhea: Advances in pathogenesis and management. Obstet Gynecol 108: 428-441, 2006. 